\title{
Inactivation of Sox9 in fibroblasts reduces cardiac fibrosis and inflammation
}

\author{
Gesine M. Scharf, ${ }^{1,2}$ Katja Kilian, ${ }^{1}$ Julio Cordero, ${ }^{3}$ Yong Wang, ${ }^{1,4}$ Andrea Grund,,${ }^{1,2}$ Melanie Hofmann, ${ }^{1}$ \\ Natali Froese, ${ }^{1}$ Xue Wang, ${ }^{1}$ Andreas Kispert, ${ }^{5}$ Ralf Kist, ${ }^{6}$ Simon J. Conway, ${ }^{7}$ Robert Geffers, ${ }^{8}$ \\ Kai C. Wollert,, ${ }^{1,4}$ Gergana Dobreva, ${ }^{3,9}$ Johann Bauersachs, ${ }^{1}$ and Joerg Heineke ${ }^{1,2,9}$ \\ 'Department of Cardiology and Angiology, Hannover Medical School, Hannover, Germany. ${ }^{2}$ Department of Cardiovascular \\ Research and ${ }^{3}$ Department of Anatomy and Developmental Biology Center for Biomedicine and Medical Technology \\ Mannheim, European Center for Angioscience, Medical Faculty Mannheim of Heidelberg University, Mannheim, Germany. \\ ${ }^{4}$ Division of Molecular and Translational Cardiology, Department of Cardiology and Angiology, and ${ }^{5}$ nstitute of Molecular \\ Biology, Hannover Medical School, Hannover, Germany. ${ }^{6}$ Institute of Genetic Medicine, Faculty of Medical Sciences, and \\ Centre for Oral Health Research, School of Dental Sciences, Newcastle University, Newcastle upon Tyne, United Kingdom. \\ ${ }^{7} \mathrm{HB}$ Wells Center for Pediatric Research, Indiana University School of Medicine, Indianapolis, Indiana, USA. ${ }^{8} \mathrm{Helmholtz}$ \\ Center for Infection Research, Braunschweig, Germany. ${ }^{9}$ German Center for Cardiovascular Research partner site \\ Mannheim/Heidelberg, Heidelberg, Germany.
}

Fibrotic scarring drives the progression of heart failure after myocardial infarction (MI). Therefore, the development of specific treatment regimens to counteract fibrosis is of high clinical relevance. The transcription factor sex-determining region $\mathrm{Y}$ box 9 (SOX9) functions as an important regulator during embryogenesis, but recent data point toward an additional causal role in organ fibrosis. We show here that SOX9 is upregulated in the scar after MI in mice. Fibroblast-specific deletion of Sox9 ameliorated MI-induced left ventricular dysfunction, dilatation, and myocardial scarring in vivo. Unexpectedly, deletion of Sox9 also potently eliminated persisting leukocyte infiltration of the scar in the chronic phase after MI. RNA-Seq from the infarct scar revealed that Sox 9 deletion in fibroblasts resulted in strongly downregulated expression of genes related to extracellular matrix, proteolysis, and inflammation. Importantly, Sox9 deletion in isolated cardiac fibroblasts in vitro similarly affected gene expression as in the cardiac scar and reduced fibroblast proliferation, migration, and contraction capacity. Together, our data demonstrate that fibroblast SOX9 functions as a master regulator of cardiac fibrosis and inflammation and might constitute a novel therapeutic target during MI.

Authorship note: GMS and KK contributed equally to this work.

Conflict of interest: The authors have declared that no conflict of interest exists.

Copyright: (c) 2019, American Society for Clinical Investigation.

Submitted: December 10, 2018

Accepted: July 9, 2019

Published: July 16, 2019.

Reference information: JCI Insight. 2019;4(15):e126721.

https://doi.org/10.1172/jci.

insight.126721.

\section{Introduction}

Chronic heart failure ( $\mathrm{CHF}$ ) emerges most commonly after myocardial infarction (MI) in the Western world (1). Despite some recent advances in treatment strategies, the mortality of patients with $\mathrm{CHF}$ remains unacceptably high. Cardiac fibrosis is a major driver of disease progression in CHF (2). Although the deposition of extracellular matrix (ECM) is needed to replace dead cardiomyocytes after $\mathrm{MI}$ and to prevent ventricular rupture, excessive fibrosis causes large infarct scars resulting in cardiac dilatation and reduced cardiac function (2-4). Within the first days after MI, scar formation coincides with the reduction of leukocyte infiltration and inflammation, which is initially needed to clear necrotic cardiomyocytes (5). Adequate termination of the inflammatory response, however, is required in the myocardium to prevent left ventricular dilatation, which (besides large MI size) is the main contributor to heart failure development and poor prognosis (6)

Although initial infarct size after MI can be therapeutically reduced by immediate percutaneous coronary interventions to restore patency of the infarcted coronary vessel, ECM deposition and related scar expansion as well as persisting myocardial inflammation cannot be targeted by current therapies $(6,7)$. In this regard, it would be especially desirable to modulate myocardial scar tissue in a way that reduces matrix deposition and inflammatory signals. However, neither the predominantly responsible cell type(s) nor lineage-restricted nodal molecular regulators that could be addressed by future therapies are well defined. 
Resident cardiac fibroblasts are considered the main homeostatic producer of ECM in the heart, although other cells, for example cardiomyocytes, are also able to make and secrete collagens (8). In response to pathological overload or ischemic injury, quiescent fibroblasts become activated, proliferate, migrate, and differentiate toward a pathological myofibroblast phenotype, which deposits excess ECM and exerts contractile properties (8).

Sex-determining region Y box 9 (SOX9) is a transcription factor that plays an essential role during mammalian development, where it crucially regulates chondrogenesis and sex differentiation (9-12). Heterozygous mutations of the $S O X 9$ gene lead to the phenotype of campomelic dysplasia, a human skeletal malformation syndrome with XY sex reversal that is usually lethal within the neonatal period $(13,14)$. As an important regulator of ECM genes, SOX9 plays a significant role in the pathogenesis of various fibrotic diseases, such as liver fibrosis, glomerulosclerosis, and heart valve calcification (15-17). Recently, SOX9 was suggested for the first time as a potential regulator of cardiac fibrosis in mice after ischemia/reperfusion injury (18). The functional relevance of SOX9 in pathological cardiac fibrosis, the cardiac cell type it acts in to promote ECM deposition, as well as its effect on the entire fibroblast transcriptome, however, remain currently unknown.

Here, we describe that fibroblast-specific downregulation of Sox 9 reduces ECM deposition in a mouse model of $\mathrm{MI}$ in vivo and that it prevents the activation toward a proliferative and migrating fibroblast phenotype in cell culture in vitro. In addition, we demonstrate that reduced Sox9 expression in fibroblasts ameliorates persisting inflammation within the infarct scar, prevents cardiac dilatation, and improves cardiac function after MI.

\section{Results}

Sox9 is expressed in cardiomyocytes and cardiac fibroblasts and is upregulated after MI in mice. Immunofluorescence staining for SOX9, platelet-derived growth factor receptor- $\alpha$ (PDGFR- $\alpha$, a marker for cardiac fibroblasts) (19), and cardiac troponin T (as a cardiomyocyte marker) showed expression of SOX9 in nuclei of cardiac fibroblasts and cardiomyocytes in adult mouse ventricles (Figure 1A). Quantification of mRNA levels in isolated cardiomyocytes and isolation of cardiac fibroblasts by quantitative PCR (qPCR) from uninjured mouse hearts confirmed Sox 9 expression in both cell types, although mRNA levels of Sox 9 were markedly higher in fibroblasts compared with cardiomyocytes (Supplemental Figure 1A; supplemental material available online with this article; https://doi.org/10.1172/jci.insight.126721DS1). Analysis of SOX9 by immunoblotting showed a significant upregulation of SOX9 in the infarct zone early after MI (day 3), which partially decreased over time in the scar region 7 and 28 days after MI but remained significantly elevated compared with uninjured sham hearts or the remote zone after MI. In contrast, ventricular samples from the remote area of the same MI-injured mice showed no significant difference in SOX9 protein levels compared to sham-operated mice (Figure 1, B-F).

To investigate the functional relevance of Sox9 in activated fibroblasts in vivo, B6;129Sv-Sox $9^{\text {tmlGst }}$ $\left(\right.$ Sox $\left.9^{f / f}\right)$ mice were bred with mice expressing Cre recombinase linked to the periostin (Postn) promoter to obtain mice with specific Sox9 knockout in activated fibroblasts (Postn ${ }^{\mathrm{Cre} /+}$ Sox $9^{f / f l}$; for short, Sox9fl-PerCre; Figure 2A). Postn is expressed in various structures under normal conditions during cardiac development (e.g., in the developing cardiac cushions), but its expression in quiescent fibroblasts or cardiomyocytes of the uninjured adult heart is negligible. However, the Postn-Cre allele is robustly upregulated in adult activated fibroblasts following cardiac injury (20-24). Accordingly, immunofluorescence staining of SOX9 in the infarct area of Sox9f-Per-Cre mice 7 days after MI revealed a marked reduction of SOX9+ fibroblasts (stained with PDGFR- $\alpha$ ) compared with littermate controls (Sox $9^{f / f l}$; for short, Sox $9^{f / f l}$; see Figure 2, B and C). Moreover, immunoblot analysis of Sox9fl-Per-Cre mice after MI revealed a marked reduction of SOX9 protein levels compared with control mice only in the cardiac scar but not in the remote region of the myocardium (Figure 2, D-F). Because the scar is composed mainly of fibroblasts with only a few residual myocytes, these results suggested decreased expression of SOX9 in fibroblasts within the scar region after MI, but not in the remote area, which predominantly (at least with regard to cell volume) consists of cardiomyocytes. Hence, Sox9fl-Per-Cre mice exerted reduced fibroblast SOX9 levels within the scar but not in the cardiomyocyte compartment in the remote myocardium.

Fibroblast-specific Sox9 deletion improves cardiac function after MI. To analyze the impact of fibroblast SOX9 after MI, Sox9fl-Per-Cre and Sox $9^{f / f l}$ (control mice) underwent left anterior descending coronary artery (LAD) ligation or sham surgery. After 42 days, we found no difference in heart weight/body 
A

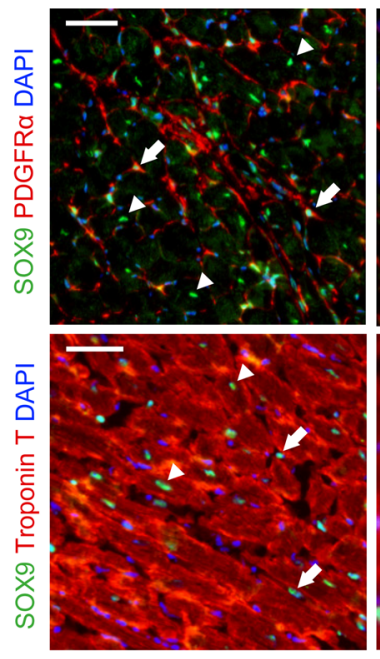

C

3 days after $\mathrm{MI}$



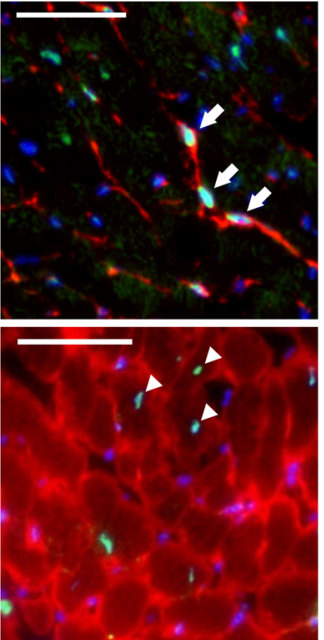
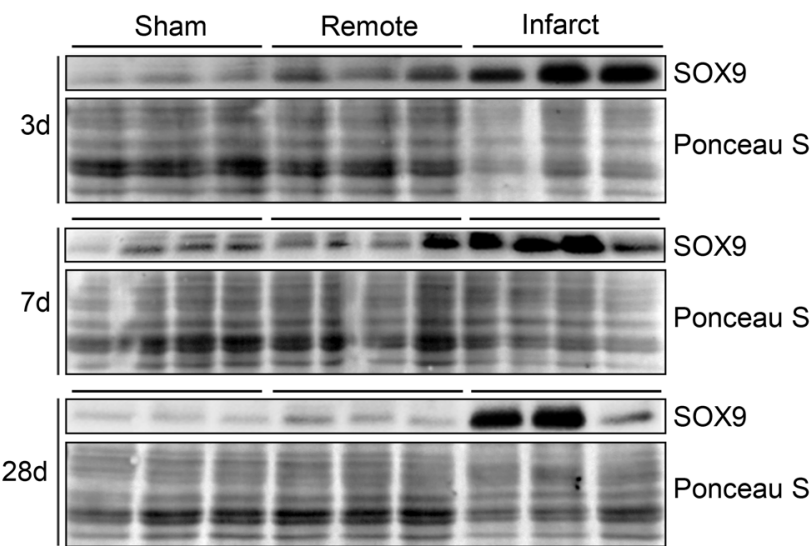

E
28 days after MI

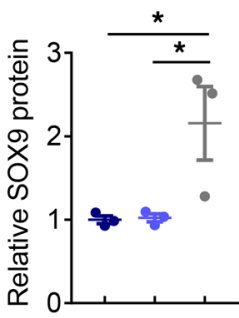

F Time course of SOX9 in the infarct region after MI

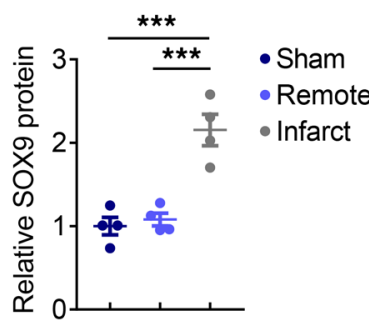

- Sham

- Remote

- Infarct

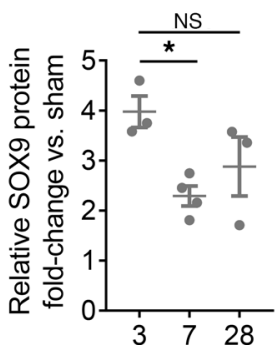

Figure 1. Cardiac S0X9 protein level after MI. Adult wild-type mice underwent MI by permanent ligation of the left anterior descending artery. (A) Representative immunofluorescence images show SOX9 (green) in cardiac fibroblasts (stained with PDCFR- $\alpha$, red) and cardiomyocytes (stained with troponin T, red) in the remote area; nuclei are stained with DAPI (blue). Arrows indicate SOX9+ cardiac fibroblasts; arrowheads indicate SOX9+ cardiomyocytes. Scale bar: $50 \mu \mathrm{m}$. (B-F) Western blot analysis (B) and densitometric quantification (C-F) of SOX9 protein level in heart tissue of the remote or infarct area and sham controls isolated from wild-type mice 3, 7, or 28 days after sham operation or MI. Ponceau S (range 40-55 kDa) was used as a loading control. For comparison of SOX9 protein level in the infarct area at different time points $(\mathbf{F})$, data were normalized to 3 -day sham as a reference. Data are shown as mean \pm SEM. $n=3-4$ mice/group; 1-way ANOVA with Tukey's multiple-comparisons test; ${ }^{*} P<0.05,{ }^{* * *} P<0.001,{ }^{* * * *} P<0.0001$.

weight (HW/BW) ratio between Sox9f-Per-Cre and control mice, while it significantly increased in both genotypes after MI versus sham-operated mice (Figure 3A). The lung weight/body weight (LW/BW) ratio was not different between Sox9fl-Per-Cre and control mice, and there was no difference between the MI and sham cohorts, indicating that the animals were in a compensated state of heart failure without pulmonary edema (Figure 3B). Transthoracic echocardiography revealed a significantly better systolic cardiac function (measured as left ventricular ejection fraction) of the Sox9fl-Per-Cre compared with control mice 7 days after MI, which was maintained until 42 days after surgery (Figure 3, C and D). Moreover, the left ventricular chambers of Sox9fl-Per-Cre mice were significantly less dilated (i.e., exerted a reduced LVEDA) 7 and 42 days after MI compared with control mice (Figure 3, E and F). No difference occurred between both genotypes at any time point after sham surgery, and no anatomical abnormalities were observed in Sox9fl-Per-Cre mice.

Deletion of Sox9 in fibroblasts in vivo reduces myocardial scarring and fibroblast proliferation after MI. To identify the underlying cause for the preservation of cardiac function after MI upon Sox9 deletion in fibroblasts, we quantified the extent of myocardial scar formation in hearts 7 days after MI. Masson's trichrome staining revealed significantly smaller myocardial scar areas in Sox9fl-Per-Cre compared with control mice (Figure 4, A and B). Immunofluorescence staining for the proliferation marker Ki67 with PDGFR- $\alpha$ as a fibroblast marker suggested reduced fibroblast proliferation in the scars of Sox9fl-Per-Cre compared with control mice 7 days after MI (Figure 4, C and D). To investigate whether 
A

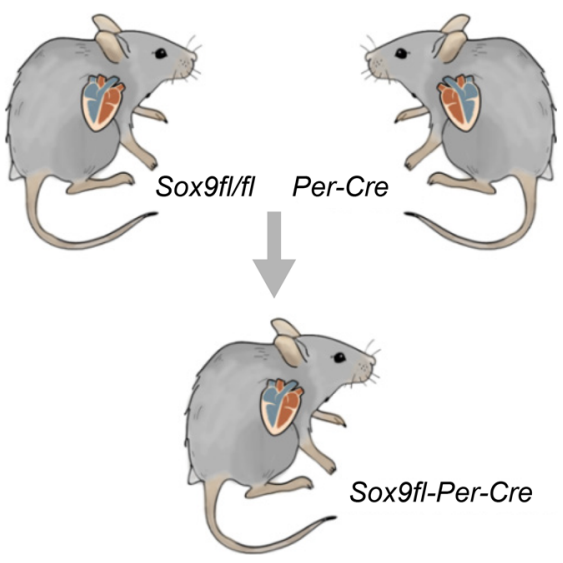

D
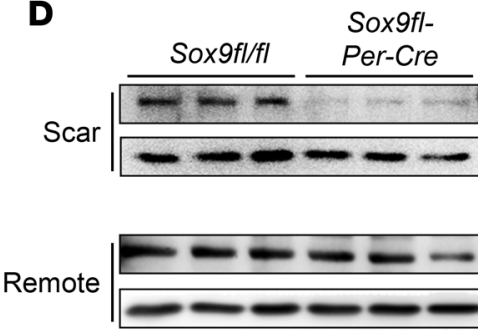

B

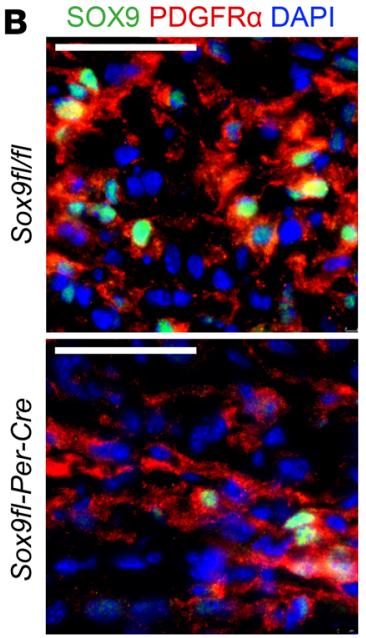

E

SOX9

SOX9

GAPDH

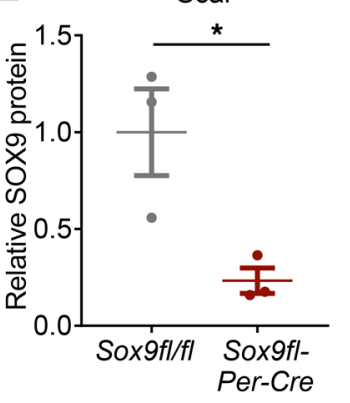

C

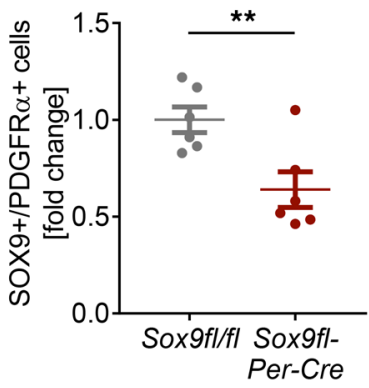

$\mathbf{F}$

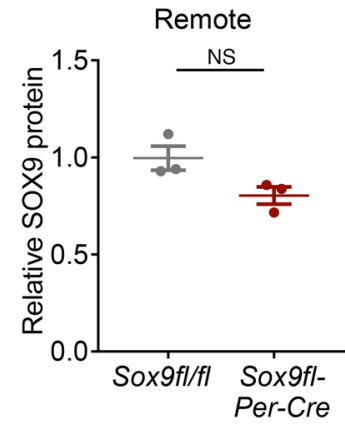

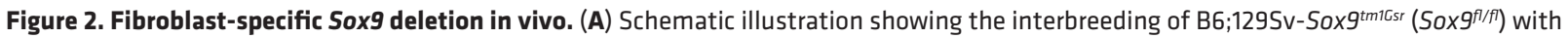
$\mathrm{Tg}$ (Postn-Cre)1Sjc (Per-Cre) mice to obtain mice with specific Sox9 deletion in activated cardiac fibroblasts (Sox9fl-Per-Cre). (B and C) Representative IHC images (B) and quantification (C) of SOX9+ fibroblasts from the scar area of Sox fl/fl $^{\text {and Sox } 9 f-P e r-C r e ~ h e a r t ~ t i s s u e ~} 7$ days after MI by permanent ligation of the left anterior descending artery showing SOX9 (green) in cardiac fibroblasts (stained for PDCFR- $\alpha$, red); nuclei are stained with DAPI (blue). Scale bars: $50 \mu \mathrm{m}$. A total of $n=6$ hearts per group were stained, and at least 4 images per heart were analyzed for C. (D-F) Western blot analysis (D) and densitometric quantification (E and $\mathbf{F}$ ) illustrating a highly efficient deletion of SOX9 protein from Sox9fl-Per-Cre mouse cardiac scar tissue 42 days after MI. SOX9 protein levels in remote samples after MI show no significant differences. GAPDH was used as a loading control. Data are shown as mean \pm SEM. $n=$ 3 mice/group. Two-tailed Student's $t$ test was used for comparison of 2 groups; ${ }^{*} P<0.05,{ }^{* *} P<0.01$.

Sox9 deletion prevents the formation of myofibroblasts, we performed immunofluorescence staining for smooth muscle actin ( $\alpha$-SMA) in infarct hearts of Sox9fl-Per-Cre compared with control mice. We found a clear increase of $\alpha$-SMA deposition in the infarct area of Sox9fl-Per-Cre mice as well as littermate controls compared with the remote zone, with a trend toward higher $\alpha$-SMA deposition in the infarct area of control mice, although a high variability of staining intensity existed within the scars and between mice (Figure 4E). Quantification of Acta2 mRNA level in the remote and infarct area of Sox9fl-Per-Cre and control mice 7 days after MI confirmed the increase of Acta2 in the infarct area compared with control. Although there was a trend toward lower Acta2 mRNA level in the infarct tissue of Sox9fl-Per-Cre compared with control mice, we did not find a significant reduction of Acta2 mRNA levels upon Sox9 deletion in our in vivo model (Figure 4F).

Deletion of Sox9 in vitro reduces proliferation, migration, and gel contraction of cardiac fibroblasts but has no direct effect on $\alpha$-SMA formation. To investigate the direct effects of Sox 9 deletion on the phenotype of cardiac fibroblasts, we extracted fibroblasts from hearts of adult Sox $9^{7 / f}$ mice and treated them with adenoviruses expressing Cre recombinase (AdCre) or control virus (Ad $\beta$-gal). qPCR and Western blotting revealed highly efficient downregulation of Sox 9 mRNA (by about $90 \%$ ) and protein expression (by about 60\%) in AdCre-treated fibroblasts in vitro (Supplemental Figure 1B and Figure 5, A and B). Cell proliferation was assessed with a BrdU incorporation immunoassay, which revealed significantly less proliferation in Sox9-deficient fibroblasts compared with control cells (Figure 5C), thereby confirming the in vivo results shown in Figure 4, C and D. To measure the fibroblast contractile capacity, which is a hallmark of cell differentiation into myofibroblasts, the cells were infected with AdCre or Adß-gal and 
A



C

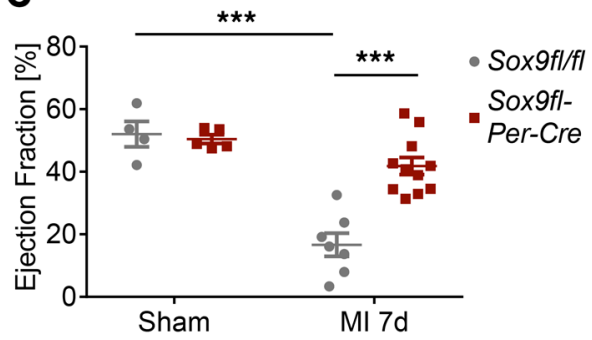

E

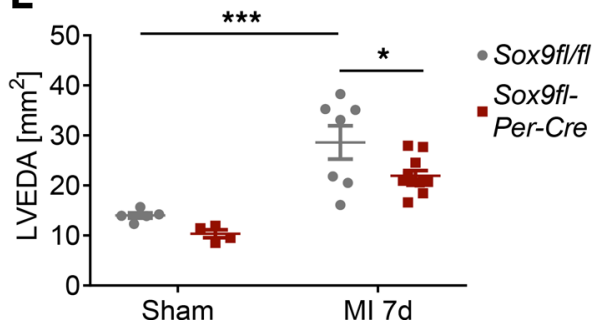

B

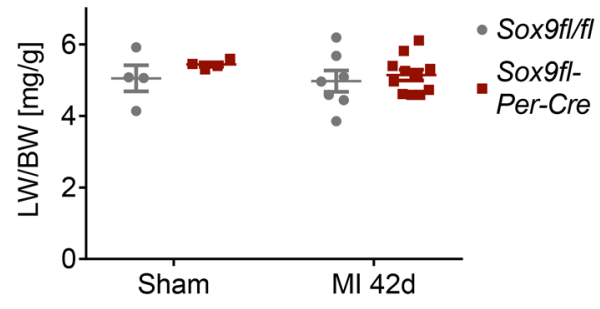

D
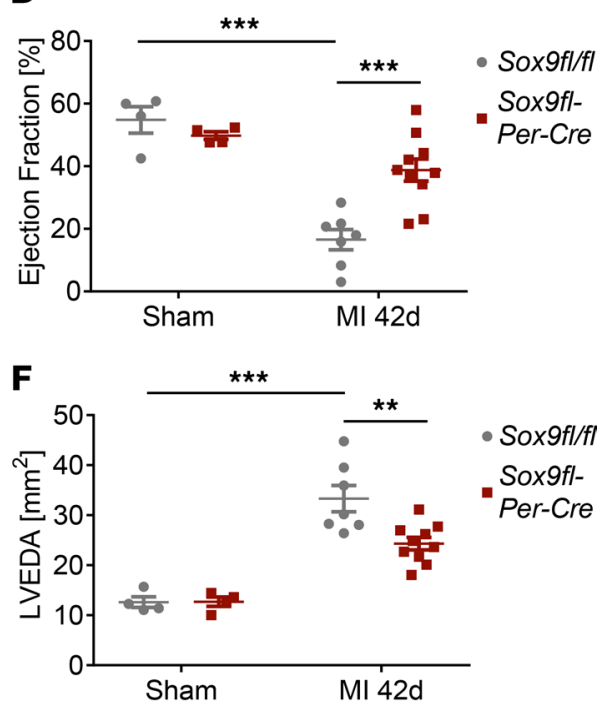

Figure 3. Improved cardiac function of mice with fibroblast-specific Sox $\mathbf{9}$ deletion after MI. Morphological analysis and functional evaluation of Sox $9^{f / f l}$ and Sox9fl-Per-Cre mice 7 days and 42 days after MI by permanent ligation of the left anterior descending artery. (A and B) Quantification of the HW/BW ratio (A) shows an increase in Sox $9^{f / f l}$ and Sox9fl-Per-Cre mice 42 days after MI; the LW/BW ratio (B) shows no significant differences. (C-F) Echocardiography showed an improved ejection fraction in Sox9fl-Per-Cre mice compared with Sox $9^{f / f l}$ mice 7 (C) and 42 (D) days after MI. Moreover, Sox9fl-Per-Cre mice exerted less left ventricular dilatation, i.e., increased left ventricular end-diastolic area (LVEDA), in relation to Sox $9^{f / f l}$ mice 7 (E) and 42 (F) days after MI. Sham-operated mice had no impairment of cardiac

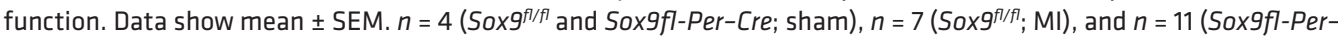
Cre; MI). Two-way ANOVA with Holm-Šídák's multiple-comparisons test; ${ }^{*} P<0.05,{ }^{* *} P<0.01,{ }^{* * *} P<0.001$.

poured into a collagen gel matrix. After 48 hours, contraction was quantified as the difference between the gel area after 48 hours and 0 hours, which showed that gel matrices with Sox9-deficient fibroblasts were significantly less contracted (indicating less myofibroblast abundance) than the control samples (Figure 5D). Moreover, cell migration was analyzed under baseline conditions or after stimulation with angiotensin II, TGF- $\beta 1$, or FBS. The migration level, quantified as closure of a defined scratch area within 24 hours, was reduced in Sox9-deficient cardiac fibroblasts under baseline conditions as well as after stimulation with angiotensin II, TGF- $\beta 1$, or FBS (Figure 5, E-H). Overall, we found that Sox9 deficiency causes less active cardiac fibroblasts compared with control cells in vitro.

Next, we analyzed the formation of stress fibers as a key marker of myofibroblast differentiation in isolated cardiac fibroblasts with Sox9 deletion compared with control cells, under baseline conditions or after stimulation with TGF- $\beta 1$ for 24 hours to induce myofibroblast differentiation. Unexpectedly, fluorescence staining for filamentous actin with phalloidin and consecutive quantification of stress fiber-positive fibroblasts failed to reveal differences between Sox9-deficient cardiac fibroblasts and control cells (Supplemental Figure 1, C and D). Moreover, mRNA levels of key myofibroblast differentiation markers, particularly Acta2 and others (Fmn1, Cnn1, Tagln), were similarly expressed between Sox9-deficient cardiac fibroblasts and control cells (Supplemental Figure 1, E-H).

Fibroblast SOX9 controls ECM formation and inflammation in the chronic stage after MI in vivo. Next, we investigated the effect of fibroblast-specific Sox 9 deletion in the late phase after MI when the formation of the chronic scar is completed and myofibroblasts are further differentiated into quiescent matrifibrocytes (25). 
A

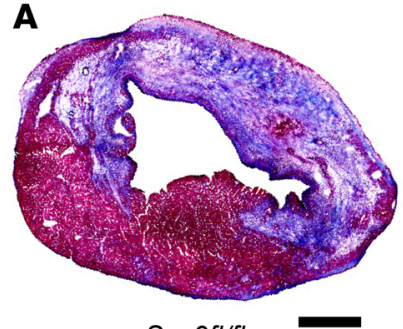

Sox9fI/fI

B



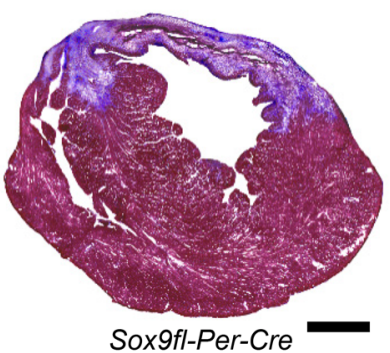

D

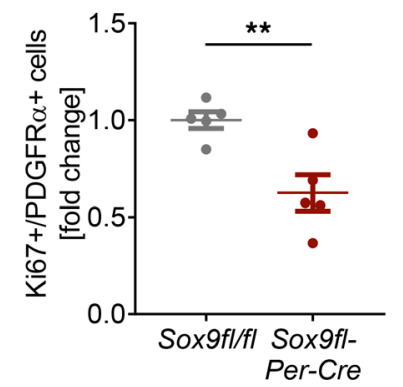

C

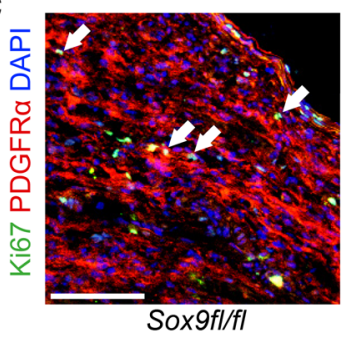

E

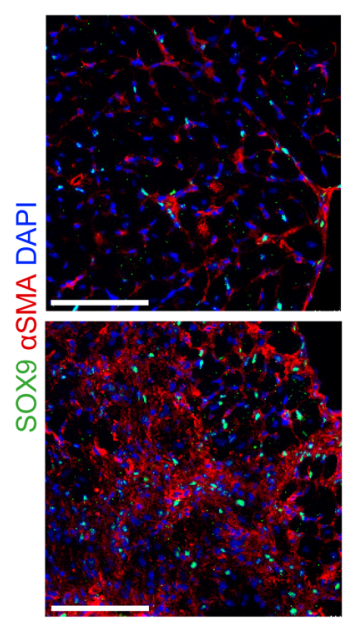

Sox9fl/fI

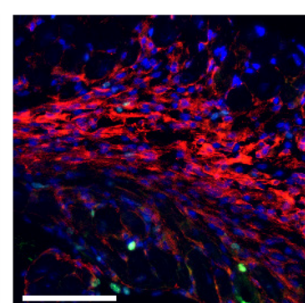

Sox9fl-Per-Cre
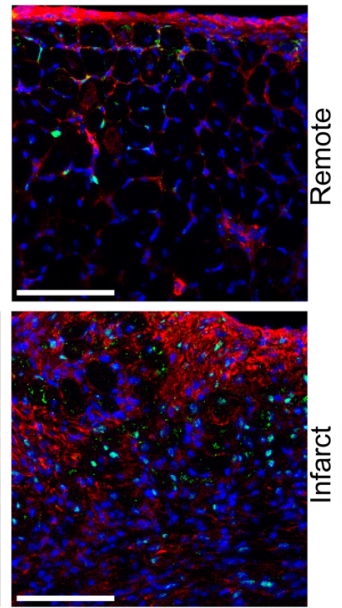

Sox9fl-Per-Cre

Figure 4. Fibroblast-specific Sox9 deletion reduces the scar area and attenuates the activation of cardiac fibroblasts after MI. (A and B) Masson's trichrome staining of representative midventricular sections $(\mathbf{A})$ and quantification of the scar area (B) of Sox9fl-Per-Cre ( $n=9)$ compared with Sox9flfl $(n=$ 7) mouse hearts 7 days after MI. Scale bars: 1 mm. (C and D) Representative IHC images showing Ki67 (green) and PDCFR- $\alpha$ (red) as markers for fibroblast proliferation in the infarct region of Sox $9^{f / f l}$ and Sox9fl-Per-Cre mouse hearts 7 days after MI (C) and quantification (D). Nuclei are shown with DAPI (blue). Arrows indicate exemplary Ki67+ fibroblasts. Scale bars: $100 \mu \mathrm{m}$. A total of 5 hearts per group were stained, and 4 images per heart were analyzed for $\mathbf{D}$. (E) Representative IHC images showing SOX9 (green) and $\alpha$-SMA (red) in the remote and infarct area of Sox $9^{f / f l}$ and Sox9fl-Per-Cre mouse hearts 7 days after MI. Nuclei are shown with DAPI (blue). Representative images of 5 hearts per group are shown. Scale bars: $100 \mu$ m. (F) mRNA expression of Acta2

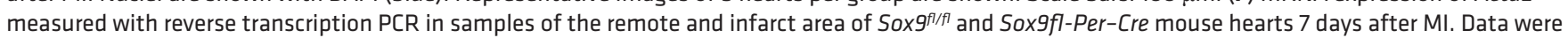
normalized to Acta2 in the remote area of Sox $9^{f / f l}$ mice 7 days after MI. $n=5$ (Sox $9^{f / f l}$ ) and 6 (Sox9fl-Per-Cre). Data are shown as mean \pm SEM. Student's $t$ test was used for comparison of 2 groups (B and D); 2 -way ANOVA with Holm-Šídák's multiple-comparisons test was used for F. $P$ value for Acta2 in the infarct area of Sox $9^{f / f l}$ and Sox9fl-Per-Cre was 0.11 (indicated as n.s. in F). ${ }^{* *} P<0.01,{ }^{* * * *} P<0.0001$.

Masson's trichrome staining of the infarcted hearts 42 days after MI revealed that the scar area of Sox9flPer-Cre mice was still significantly smaller compared with controls at this late stage (Figure 6, A and B).

To identify SOX9-dependent gene regulation in the chronic myocardial scar, we performed a genome-wide transcriptomic analysis of cardiac scar tissue from Sox9fl-Per-Cre and Sox $9^{\text {flfl }}$ mice 42 days after MI by RNA-Seq. Genes with significant differential regulation between both conditions (at least $50 \%$ up- or downregulated) are demonstrated in the heatmap (Figure 6C and Supplemental Figure 2A). In the chronic infarct scar of Sox9fl-Per-Cre mice, 2611 genes were downregulated and belonged to the GO (biological process) classes cartilage development, cell chemotaxis, extracellular matrix organization, immune response, and proteolysis (Figure 6C). Highly downregulated genes in the myocardial scar of Sox9fl-Per-Cre mice included mainly collagen genes (Col2a1, Col9a2, Col11a1), inflammatory mediators (Cxcl13, Il6, Tnfsf15), and proteases (Adam32, Prss23, Capn6; other example genes are listed in Figure 6C and in Supplemental Figure 2, B-D). In the scar of Sox9fl-Per-Cre mice, 1218 genes were upregulated, which were related mainly to oxidative cellular metabolism (GO classes ATP metabolic process, tricarboxylic acid cycle, and fatty acid metabolic process), for example, Atp $5 b$, $I d h 3 a$, and $A c a d l$, but we also found genes of the category muscle contraction, for example, Myh6, Tnni3, and Ttn (more example genes are listed in Figure 6C) within this group. We subsequently 
A

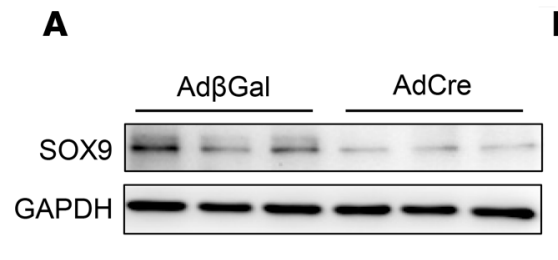

E

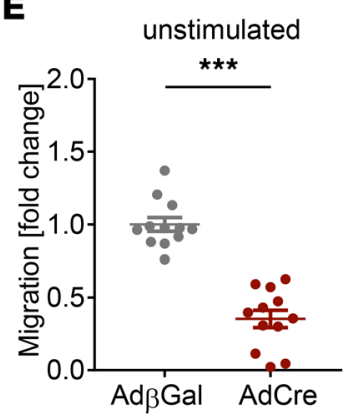

G

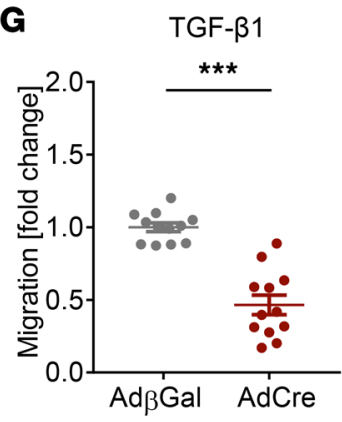

B



C

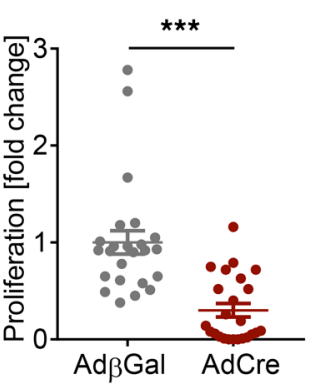

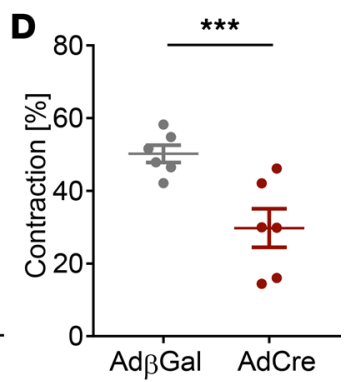

$24 \mathrm{~h}$
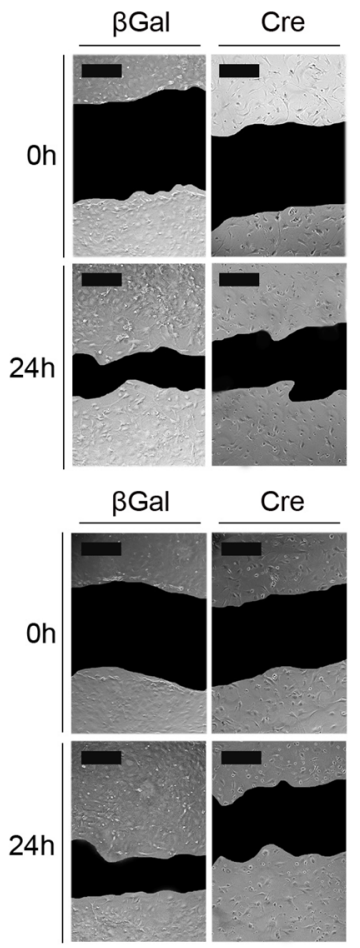

$\mathbf{F}$
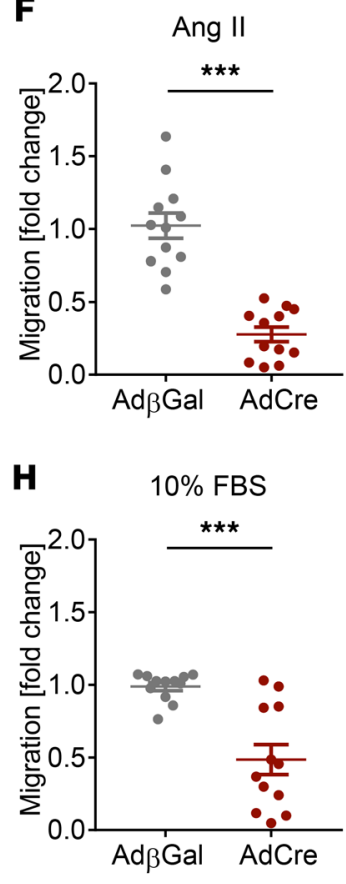
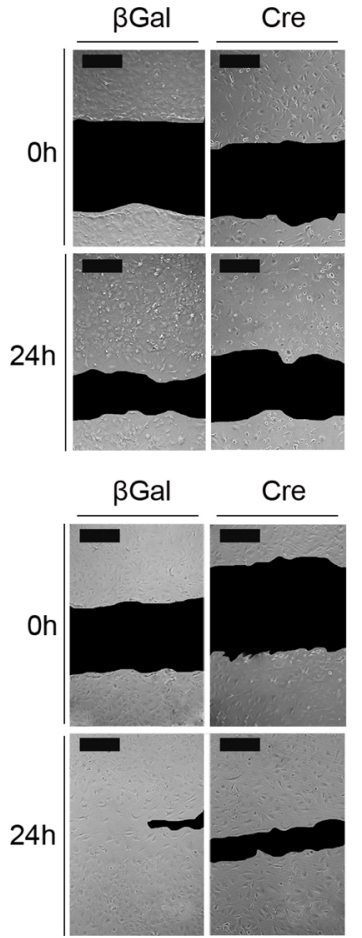

Figure 5. In vitro Sox9 deletion in cardiac fibroblasts reduces migration, proliferation, and contractility. Isolated cardiac fibroblasts from uninjured Sox $9^{\text {fl/f }}$ mouse hearts were infected with adenovirus expressing Cre recombinase (AdCre) to generate in vitro Sox 9 loss of function or control adenovirus (Ad $\beta$-gal). (A and B) Efficiency of SOX9 deletion is demonstrated on the protein level by Western blot (A) and densitometric quantification (B). (C) Proliferation of cardiac fibroblasts with Sox9 deletion compared with control cells was measured by BrdU incorporation ( $n=24$ samples/group). (D) Contraction capacity of cardiac fibroblasts quantified as area change of a collagen matrix containing fibroblasts with Sox 9 deletion or control cells after 48 hours compared with baseline at 0 ( $n=6$ samples/group). (E-H) Migration of Sox $9^{f / f l}$ fibroblasts treated with either Ad $\beta$-gal ( $\beta$-gal) or AdCre (Cre) under baseline conditions (unstimulated, E) or in the presence of profibrotic stimuli angiotensin II (Ang II, F), TGF- $\beta 1$ (C), or $10 \%$ FBS (H), quantified as scratch closure after 24 hours. Representative pictures are shown directly after scratch and 24 hours later; $n=12$ samples/group. Scale bars: $400 \mu \mathrm{m}$. Data are shown as mean \pm SEM. Two-tailed Student's $t$ test was used for comparison of 2 groups; ${ }^{* *} P<0.01 ;{ }^{* *} P<0.001$.

focused on the genes that were downregulated because of Sox9 deletion in Sox9fl-Per-Cre mice because for these genes direct regulation by SOX9 was more likely. To investigate whether these downregulated genes might be direct targets of SOX9, we intersected our RNA-Seq data with SOX9 ChIP-Seq results from developing mammalian chondrocytes (26). In that study, SOX9 binding regions were characterized throughout the genome, and SOX9 was found to bind close to the promoter in an indirect manner ("class I engagement," found mostly in genes with general cellular functions) or to bind directly to DNA in enhancer regions of cartilage-related genes, which were in large part identical to the ECM genes in our study. Intersection with the downregulated genes from our data set revealed that $50.5 \%$ of the downregulated genes exerted direct SOX9 binding, whereby $43 \%$ showed class II engagement or both class I and class II engagement. GO term analysis in these $43 \%$ of downregulated genes reflected very similar GO classes (leukocyte migration, cell chemotaxis, extracellular matrix organization; Supplemental Figure 3A) as observed among all downregulated genes in the myocardial scar of Sox9fl-Per-Cre mice. KEGG pathway analysis revealed slightly more class I engagement alone but 
A

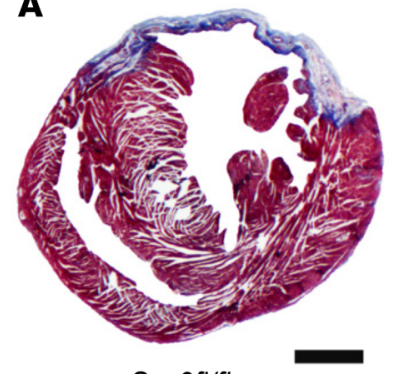

Sox $9 f I / f I$

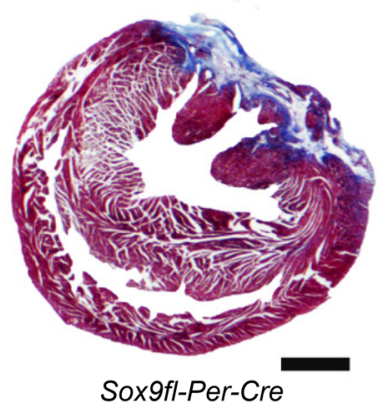

Sox9fl-Per-Cre
B

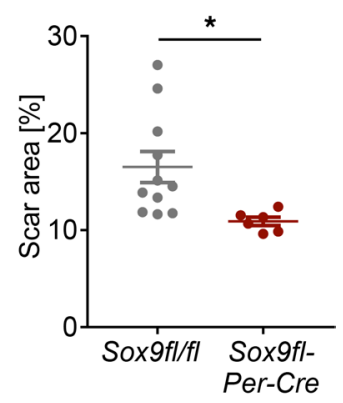

C

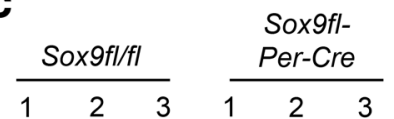

Down in Sox9fl-Per-Cre Scar: GO classification, example genes

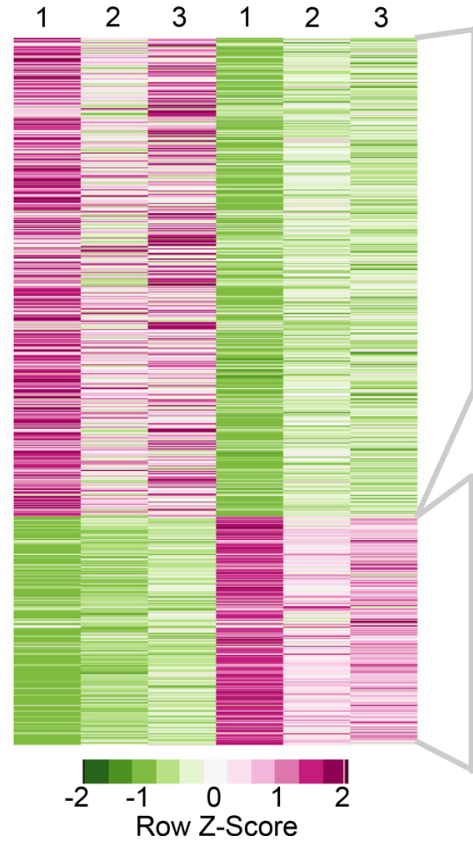

Cartilage development $\left(P=1.8^{*} 10^{-6}\right)$

Acan, Col1a1, Col2a1, Col11a1, Ctgf, Sox9, Sox5

Cell chemotaxis $\left(P=8.8^{*} 10^{-9}\right)$

Cxcl13, Ccl6, Cc/9, Ccl17, Cxcl10

Extracellular matrix organization $\left(P=8.4^{*} 10^{-7}\right)$

Acan, Col1a1, Col2a1, Col9a2, Fbln5, Lox

Immune response $\left(P=7.2^{*} 10^{-28}\right)$

Cxc/13, Cc/17, Cxc/10, Cd244, Enpp1, II6, Tnfsf15

Proteolysis $\left(1.4^{\star} 10^{-3}\right)$

Adam32, Capn6, Mmp2, Mmp19, Mmp25, Prss23, Prss57

Up in Sox9fl-Per-Cre Scar: GO classification, example genes
ATP metabolic process $\left(P=7^{\star} 10^{-10}\right)$
Atp5b, Atp5j, Atp5f1, Atp5a1, Atp5c1
Tricarboxylic acid cycle $\left(P=6.5^{\star} 10^{-23}\right)$
Aco2, Cs, Fh1, Idh2, Idh3a, Mdh2, Sdha
Fatty acid metabolic process $\left(P=2.2^{*} 10^{-17}\right)$
Acaa2, Acadl, Acadm, Acads, Cpt2
Muscle contraction $\left(P=1.2^{*} 10^{-3}\right)$
Myh6, Tnni3, Ttn, Myom1, Cacna1c

Figure 6. Fibroblast-specific Sox9 deletion reduces the scar area and inhibits expression of proinflammatory and ECM genes after long-term MI. (A and B) Masson's trichrome staining of representative midventricular sections (A) and quantification of the scar area (B) $(n=6-11)$ of Sox9fl-Per-Cre compared with Sox $9^{f / f l}$ mouse hearts 42 days after MI. Scale bars: $1 \mathrm{~mm}$. (C) Gene expression in scar area tissue of Sox9fl-Per-Cre compared with Sox $9^{f / f l}$ mouse hearts 42 days after MI was analyzed with RNA-Seq. Heatmap of differentially regulated genes in the MI scar of Sox $9^{f / f l}$ and Sox9fl-Per-Cre mice $(n=3) 42$ days after MI is shown on the left of C. Gene Ontology (CO, biological process) classification of highly regulated processes and selected example genes are demonstrated on the right. Bioinformatic analysis of RNA-Seq data is described in the Methods section. Data are shown as mean \pm SEM. Student's $t$ test was used for comparison of 2 groups; ${ }^{*} P<0.05$.

identified otherwise similar functional classes (cytokine-cytokine receptor interaction, ECM-receptor interaction, protein digestion and absorption; Supplemental Figure 3B). Supplemental Figure 4 shows exemplary results for class I and class II binding of SOX9 to selected ECM genes (Col2a1, Col9a2, and Fn1; Supplemental Figure 4, A-C), inflammatory genes (Cxcl13 and Il6; Supplemental Figure 4; D and E), and a proteolysis gene (Mmp2; Supplemental Figure $4 \mathrm{~F}$ ) in conjunction with our RNA-Seq results. These data imply a direct interaction of SOX9 with the promoter or enhancer regions of ECM, proteolysis, or inflammatory genes identified in this study.

Because RNA-Seq showed decreased expression of inflammatory mediators in the scar region of Sox9fl-Per-Cre mice, we investigated leukocyte abundance 6 weeks after MI, which was strongly diminished upon reduced fibroblast SOX9 levels both in the scar as well as in the remote area, although in the scar leukocyte abundance was generally higher in both control and Sox9f-Per-Cre mice (Figure 7, A and B). As one example of the ECM-related gene expression, we confirmed reduced COL2A1 protein abundance in the scar of Sox9fl-Per-Cre mice by Western blot and immunofluorescence staining (Figure 7, C-E). 
A CD45 WGA DAPI
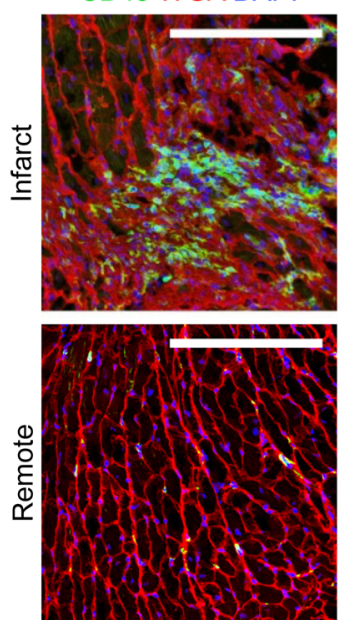

Sox9fl/fl
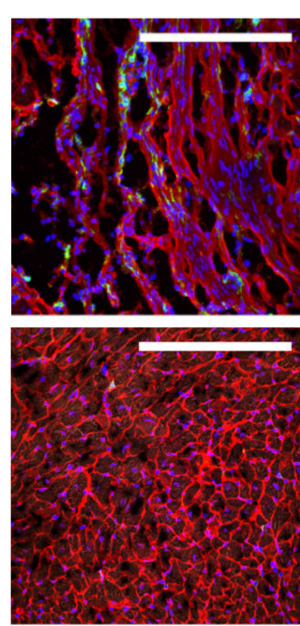

Sox9fl-Per-Cre
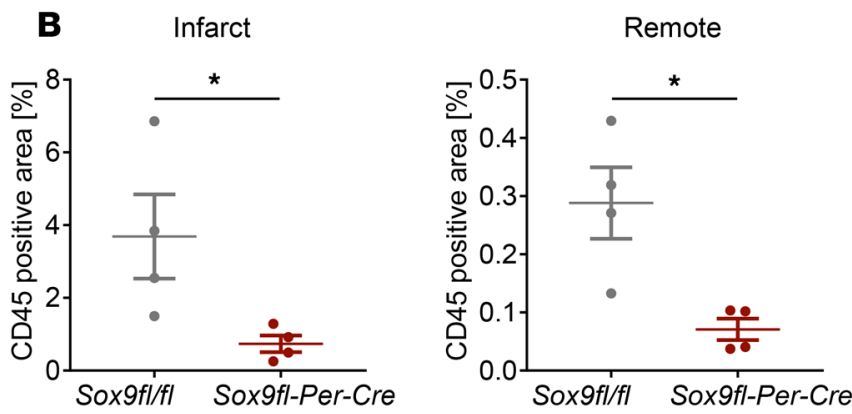

c

D

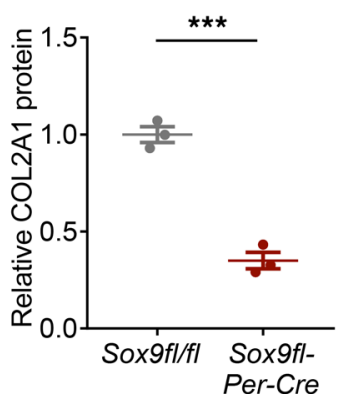

E

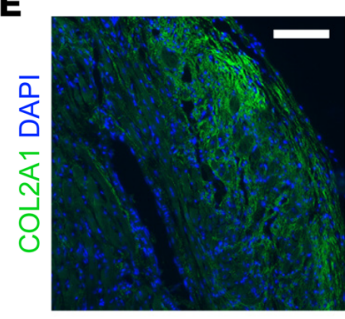

Sox9flffl

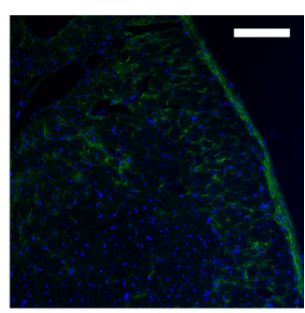

Sox9fl-Per-Cre

Figure 7. Reduced inflammation and collagen deposition after MI in mice with fibroblast-specific Sox9 deletion. (A) Representative IHC images showing CD45 (green) and wheat germ agglutinin (WCA, red) in the remote and infarct area of Sox $9^{f / f}$ and Sox9fl-Per-Cre mouse hearts 42 days after MI. (B) Quantification of the $\mathrm{CD} 45^{+}$area in IHC images of Sox $9^{f / f l}$ and Sox9fl-Per-Cre mouse hearts 42 days after MI (as representatively shown in A), confirming a significant reduction of the inflammatory response in Sox9fl-Per-Cre mouse hearts compared with Sox $9^{f / f l}$ controls 42 days after MI. Nuclei are shown with DAPI (blue). A total of 4 hearts per group were analyzed. Scale bars: $200 \mu \mathrm{m}$. (C and D) Western blot analysis (C) and densitometric quantification (D) of COL2A1 protein levels in the infarct area of Sox fflf $^{\prime / 7}$ and Sox9fl-Per-Cre mouse hearts 42 days after MI ( $n=3 /$ group). (E) Representative IHC images showing COL2A1 (green) and DAPI (blue) in Sox $9^{f / f}$ and Sox9fl-Per-Cre mouse hearts 42 days after MI. Scale bars: $200 \mu \mathrm{m}$. Data are shown as mean \pm SEM. Two-tailed Student's $t$ test was used for comparison of 2 groups; ${ }^{*} P<0.05 ;{ }^{* *} P<0.001$.

Gene expression analysis in vitro confirms that SOX9 directly controls ECM, inflammatory, and protease gene expression in isolated fibroblasts. To verify that the identified genes in vivo are indeed primarily regulated by SOX9 in cardiac fibroblasts and not any other cell types within the scar, we again extracted fibroblasts from hearts of adult Sox $9^{f / f l}$ mice and treated them with AdCre or control virus Ad $\beta$-gal as shown in Figure 5. Quantification of mRNA levels of selected ECM genes (Col2a1, Col9a2, Fn1), inflammatory mediators (Cxcl13, Ilo), and proteases (Adam32, Mmp2) confirmed their significant downregulation in fibroblasts with acute $\operatorname{Sox} 9$ deletion (Figure 8, A-C). In contrast, mRNA levels of exemplary oxidative cellular metabolism genes that were upregulated in the RNA-Seq from the myocardial scars showed no significant differences between fibroblasts treated with AdCre or control virus (Figure 8D).

\section{Discussion}

Although cardiomyocytes also produce ECM proteins and therefore can contribute to fibrosis, resident cardiac fibroblasts are currently considered the main source of scarring in the myocardium in response to ischemic injury (8). After MI, within hours, leukocytes (neutrophils and then macrophages) invade the necrotic area to clear the debris (27). With only little delay, resident fibroblasts from the border zone are activated, proliferate, and migrate into the infarcted area. Subsequently, virtually all fibroblasts transdifferentiate into myofibroblasts between days 4 and 7 after MI (25). Myofibroblasts are long-lived cells, but in the chronic phase after MI, they revert to a more quiescent "matrifibrocyte" phenotype, which expresses chondrocyte genes (25). Throughout these multiple differentiation stages, fibroblasts produce ECM to 
A

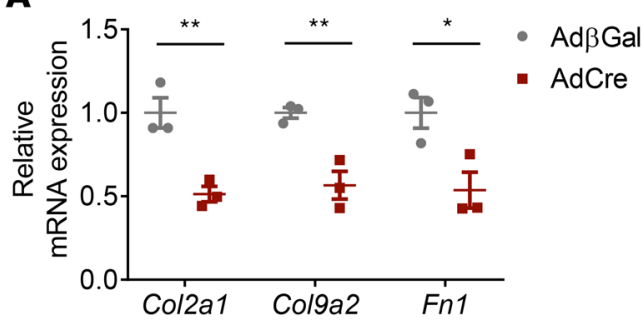

C

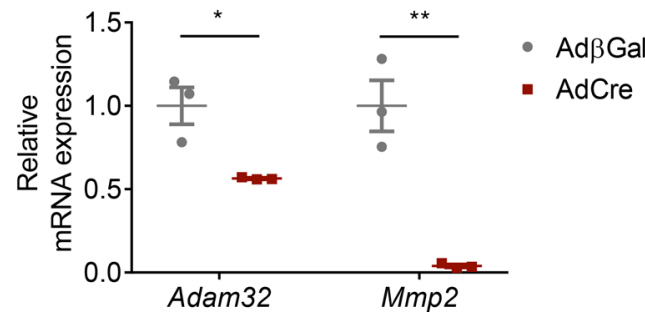

B

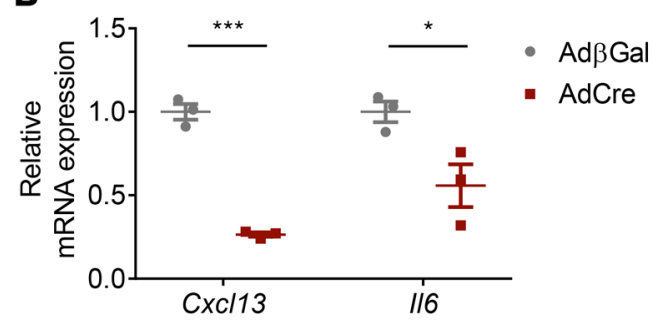

D

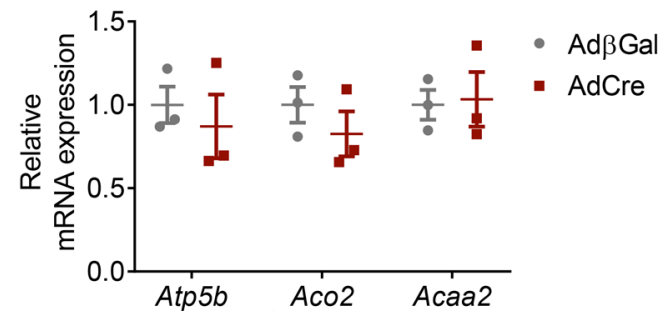

Figure 8. Sox9 deletion in isolated fibroblasts reduces expression of collagen genes, inflammatory mediators, and proteases in vitro. Isolated cardiac fibroblasts from uninjured mouse hearts of Sox $\mathrm{g}^{f / f}$ mice were infected with AdCre or Adß-gal as control to generate an efficient in vitro deletion of Sox9. (A-D) Relative mRNA expression of example genes for ECM (A), inflammatory mediators (B), proteases (C), and oxidative cellular metabolism (D) analyzed by qPCR in isolated Sox $9^{f / f l}$ cardiac fibroblasts with in vitro Sox9 loss of function or control cells. $n=3$ biological replicates measured in duplicates in each group. Data are shown as mean \pm SEM. Two-tailed Student's $t$ test was used for comparison of 2 groups; ${ }^{*} P<0.05 ;{ }^{*} P<0.01 ;{ }^{* * *} P<0.001$. Groups without indication of $P$ value were not significantly different.

generate the fibrotic scar (25). In addition, activated fibroblasts might promote inflammation, for example, in the kidney, but also in the heart, because mechanical stretching of cardiac fibroblasts in vitro promoted the expression of inflammatory mediators and might thereby help recruit monocytes, but specific in vivo evidence is scarce (28-30). Because of their versatile functions, fibroblasts might act as sentinel cells that coordinate the myocardial stress and healing response, which also renders them an ideal therapeutic target (28). Because complete myofibroblast ablation often leads to myocardial rupture, more targeted approaches will be clinically needed to counteract overactivation of fibroblasts and myofibroblasts, excessive fibrosis, and inflammation (31). In this regard, nodal upstream regulator molecules need to be defined, and we identified the transcription factor SOX9 as such a molecule.

SOX9 regulates chondrogenesis and induces fibrosis in extracardiac tissues $(32,33)$. Very recently, SOX9 was suggested to be involved in the general fibrotic response after ischemia/reperfusion injury in the adult mouse heart, although with a systemic, inducible heterozygous knockout approach, the responsible cell type was unclear, as was the functional impact of SOX9 for the heart or the SOX9-dependent general transcriptome (18). In our study, we found that SOX9 governs multiple differentiation and functional states of activated fibroblasts. Genetic SOX 9 depletion by around $60 \%$ at the protein level reduced the capability of primary isolated cardiac fibroblasts to proliferate as well as to migrate in response to multiple pathologically relevant stimuli by around $60 \%$ versus wild-type cells. In addition, reduced SOX9 level diminished the capability of fibroblasts to contract, although the formation of $\alpha$-SMA, the key marker for myofibroblasts, was not primarily affected. These in vitro data suggest that targeting of Sox 9 leads to a scalable functional response without completely ablating fibroblast function, which might be detrimental. Accordingly, genetic fibroblast-specific downregulation by a commonly used Postn-Cre approach, which mainly targets activated fibroblasts $(21,34)$, enabled a potent downregulation of SOX9 within the infarct scar. This led to a significantly reduced size of the fibrotic scar in the transition phase ( 7 days) and in the chronic phase ( 42 days) after permanent MI, indicating that SOX9 is a central regulator of scar-related fibrosis during ischemic injury. In this regard and in line with our in vitro data, fibroblasts in the scar of Sox9f-Per-Cre mice exerted less proliferative activity, which contributed to a decreased scar size. Differentiation into contractile myofibroblasts, in contrast, was only marginally affected by the lack of SOX9. Importantly, reduced fibroblast SOX9 levels potently entailed a strong, marked improvement of systolic heart function almost up to the level of sham-operated mice, which was evident as early as 7 days after MI surgery, and this improved function was maintained until 42 days after MI when the experiment was ended. Improved function was accompanied by reduced left ventricular dilation at 7 and 42 days after MI. 
To obtain insights into the SOX9-dependent transcriptome in cardiac fibroblasts, we conducted RNASeq of myocardial scar tissue 6 weeks after MI in mice with and without fibroblast-specific Sox 9 deletion. The transcriptomic analysis was therefore conducted in the chronic phase after MI, when a steady state was reached in the scar and myofibroblasts would have reached the matrifibrocyte stage. This might explain why we did not identify prominent differential regulation of cell cycle genes, because fibroblast proliferation occurs only between day 2 and day 7 after MI (25). An unexpected finding of our analysis was the upregulation of genes related to oxidative cellular metabolism upon downregulation of SOX9. Because we could not confirm this in vitro in isolated fibroblasts, we suggest that it might be a secondary phenomenon due to a higher amount of residual cardiomyocytes (which are typically characterized by active oxidative and high fatty acid metabolism) in the scar tissue of the Sox9fl-Per-Cre mice. Our hypothesis of more residual cardiomyocytes is supported by a significantly higher number of muscle contraction genes (e.g., cardiomyocyte-specific genes, such as Myh6, Tnni3, and Ttn) in MI scars with fibroblast SOX9 depletion. Among the downregulated genes and functional gene classes in the scar of Sox9fl-Per-Cre mice, of which we confirmed exemplary genes in vitro in isolated, SOX9-depleted fibroblasts, were several ECM-related genes that are known direct target genes of SOX9, for example, Acan, Col1a1, Col2a1, and Col11a1, as well as the typical chondrocyte-related genes that were previously determined as marker genes for the matrifibrocyte phenotype (Comp, Chad, Cilp2) $(25,32)$. For these genes, we also inferred binding of SOX9 to promoter and enhancer regions because of the SOX9 ChIP-Seq analysis in chondrocytes that we intersected our data with (26). This reduced expression of ECM genes contributes to reduced fibrosis, reduced scar size, and thereby also reduced cardiac dilation after MI (6). In addition, we identified downregulated expression of proteolysis enzyme genes, which in part were directly bound by SOX9 (at an adjacent enhancer in a class II dependent manner; this applies to $M m p 2,-12,-14,-16$, and -19), while others might also be expressed in leukocytes within the scar. Reduced proteolytic activity will promote the compaction of the scar and thereby reduce cardiac dilation (35). Previous work demonstrated that proteases, including MMP-2, as well as the ECM glycoprotein fibronectin, are involved in the activation of TGF- $\beta$ by cleavage from the latent TGF- $\beta$ complex and thereby indirectly influence fibroblast activation $(36,37)$. Although we did not observe any direct effects of Sox 9 deletion on the Acta2 expression or stress fiber formation in isolated fibroblasts in vitro, we observed a trend toward reduced $\alpha$-SMA staining and Acta2 mRNA levels in the infarct area of Sox9f-Per-Cre mice compared with controls 7 days after $\mathrm{MI}$ in vivo, which could have been an indirect effect of modified matrix composition with reduced fibronectin and metalloproteinase levels in the scar upon SOX9 deletion.

Very interesting and striking to us was the fact that inflammatory mediators constituted the most significantly enriched functional gene class among the SOX9-dependent downregulated genes. Several of these genes ( $C x c l 13 ; C c l 5,-6,-8$, and $-17 ; 1116$; $\operatorname{Tnfsf} 8$ ) were found to be directly bound by SOX9 at enhancer regions in chondrocytes, implying that SOX9-dependent regulation of inflammation might directly occur in fibroblasts. The direct regulation of these proinflammatory mediators through SOX9 is further supported by the fact that downregulation of $\operatorname{Sox} 9$ in isolated fibroblasts also strongly reduced the mRNA level of these genes, excluding any matrix-related secondary effects or influences of other cell types. Among these factors, Cxcl13, for example, is released by tumor fibroblasts to promote disease (38). Because of the RNA-Seq results, we analyzed leukocyte abundance and surprisingly found at this late stage (6 weeks) after MI that compared with the remote area, the scar still contained around 10-fold more leukocytes. This means that even in this "steady-state" phase, relatively high inflammatory activity exists in the scar that will likely promote cardiac dilation and disease progression and that should be therapeutically targeted. Hence, our finding that fibroblast-specific ablation of Sox9 strongly abrogates this late inflammation in the infarct scar might be of high clinical relevance in the future. Certainly, more studies will be needed before translation toward clinical medicine can be envisioned: besides identification of therapeutic tools to manipulate SOX9, it will be important to identify upstream signals that activate SOX9 and to identify its role in cardiomyocytes.

\section{Methods}

Experimental animals. To obtain mice with fibroblast-specific Sox9 deletion, previously described Sox $9^{\text {t/fl }}$ mice (B6;129Sv-Sox $9^{m 1 G s r}$ ) (39) were interbred with mice expressing Cre recombinase linked to a partial Postn promoter, $\mathrm{Tg}$ (Postn-Cre) $1 \mathrm{Sjc}$ (40). Littermate Sox $9^{\text {fl/fl}}$ mice were used as controls.

MI. MI was induced in 8- to 10 -week-old mice by ligation of the LAD, following standard procedures (41). Mice were subcutaneously injected with $0.02 \mathrm{mg} / \mathrm{kg}$ atropine and $0.1 \mathrm{mg} / \mathrm{kg}$ buprenorphine. Anesthesia was induced with $3 \%-4 \%$ isoflurane in an induction chamber. After oral intubation, mice 
were connected to a small animal ventilator (MiniVent Type 845, Harvard Apparatus), and anesthesia was maintained with $1.5 \%-2 \%$ isoflurane. A left thoracotomy was performed, and the LAD was ligated. In sham-operated control mice, the ligature around the LAD was not tied. During surgery, the animals were placed on a heating pad connected to a temperature controller (Föhr Medical Instruments) to maintain body temperature at $37^{\circ} \mathrm{C}$. To evaluate mouse survival rate, operated mice were observed daily for 42 days. We had a postprocedural mortality rate of $14.6 \%$ in the MI groups while there was no mortality observed in sham-operated mice.

Transthoracic echocardiography. Mice were anesthetized with $4 \%$ isoflurane in an induction chamber (built in-house at Hannover Medical School) and placed on a heating pad to keep body temperature at $37^{\circ} \mathrm{C}$. Anesthesia was maintained with $1 \%-2 \%$ isoflurane via mask. Noninvasive echocardiography was performed in parasternal long and short axes with a $30-\mathrm{MHz}$ transducer (Vevo 770, Visualsonics), as previously described (41).

Immunofluorescence and histological analysis. For organ harvesting, mice were sacrificed, and whole hearts were immediately removed, washed in cold PBS, and dissected at the midventricular level. Apical parts of the ventricle (distal to the LAD ligature after MI) were embedded in paraffin or Tiisue-Tek OCT Compound (Sakura).

Immunofluorescence staining was performed in 7- $\mu \mathrm{m}$ OCT cryosections. Slides were fixed in $4 \%$ PFA for 20 minutes, permeabilized in $0.3 \%$ Triton X-100 for 20 minutes, and blocked with 3\% BSA for at least 1 hour at room temperature. Each step was followed by repeated washing in PBS. Slides were then serially incubated with primary and secondary antibodies. The following primary antibodies were used for immunostaining: antiSOX9 (1:200, Merck, AB5535), anti-collagen type II (1:20, R\&D Systems; AF3615), anti-PDGFR- $\alpha$ (1:40, R\&D Systems, AF1062), anti-cardiac troponin T (1:200, Abcam, ab8295), anti-CD45 (1:50, BD Biosciences, 550539), and anti-Ki67 (1:100, Abcam, ab15580). Secondary antibodies were used in a 1:200 dilution, Alexa Fluor 488 or 555 antimouse, antirabbit, or antigoat according to the primary antibody (Cell Signaling 4409 and 4412 and Life Technologies A-21432, respectively).

Cell membrane staining was done with wheat germ agglutinin Texas red (Invitrogen, w21405) according to the manufacturer's protocol.

Staining for filamentous actin in isolated fibroblasts was done with Alexa Fluor-coupled phalloidin (Invitrogen, A12379) following the manufacturer's protocol. Slides were covered with mounting solution containing DAPI (Roche) to stain nuclei.

For light microscopic histological analysis, hearts were embedded in paraffin, and sections at 7- $\mu \mathrm{m}$ thickness were prepared. After paraffin removal, sections were stained with Masson's trichrome following standard procedures to demonstrate the scar area. The scar area was determined as the percentage of fibrotic tissue area in relation to total left ventricular area in midventricular sections (Adobe Photoshop CS6), as described previously (41).

Immunoblot analysis. For immunoblot analysis, protein lysates were prepared from frozen samples of mice hearts. Samples were then loaded on an SDS-polyacrylamide gel and subsequently blotted onto a PVDF membrane following standard procedures. Applied primary antibodies were anti-SOX9 (1:200, Santa Cruz Biotechnology, SC20095) and anti-collagen type II (1:200, R\&D Systems, AF3615). The mouse monoclonal anti-GAPDH (1:3000, Fitzgerald, 10R-G109a) or Ponceau S was used as a loading control. Western blot bands were quantified using Quantity One software.

$q P C R$. RNA was isolated using RNeasy Mini Kit (Qiagen) and the NucleoSpin RNA II kit (Macherey-Nagel) according to the manufacturers' protocols. cDNA synthesis from $1 \mu \mathrm{g}$ RNA was performed with Maxima H Minus First Strand cDNA Synthesis Kit (Thermo Fisher Scientific). SYBR Green (Thermo Fisher Scientific) on an MX4000 multiplex qPCR system (Stratagene) was used for quantitative real-time PCR. Gapdh mRNA expression levels were measured to normalize the gene expression. At least 3 replicates per group were analyzed. qPCR primers are listed in Supplemental Table 1.

Deep sequencing and bioinformatics. To identify genes regulated by SOX9 in mouse scar tissue after MI, RNA was isolated from infarct scars of Sox $9^{f / f l}$ (control) and Sox9fl-Per-Cre mice 6 weeks after MI using the RNeasy Mini Kit (Qiagen) following the instruction manual. Further analysis was performed at the Helmholtz Center for Infection Research in Braunschweig. Quality and integrity of total RNA were controlled on the Agilent Technologies 2100 Bioanalyzer. The RNA-Seq library was generated from 100 ng total RNA using Dynabeads mRNA DIRECT Micro Purification Kit (Thermo Fisher Scientific) for mRNA purification followed by ScriptSeq v2 RNA-Seq Library Preparation Kit (Epicentre) according to the manufacturers' protocols. The libraries were sequenced on Illumina HiSeq2500 using TruSeq SBS 
Kit v3-HS (50 cycles, single-ended run) with an average of $3 \times 10^{7}$ reads per RNA sample. Before alignment to the reference ( $\mathrm{mm} 10)$, each sequence was trimmed on base call quality and sequencing adapter contamination using the Trim Galore! wrapper tool (http://github.com/felixkrueger/trimgalore). Reads shorter than $20 \mathrm{nt}$ were removed from FASTQ files. Trimmed reads were aligned to the reference using the short-read aligner STAR (https://code.google.com/p/rna-star/).

Genes were quantified by the use of analyzeRepeats.pl (42) mm10 -count exons -strand both and -noadj. Differential expression analysis was performed using normalized mapped reads after trimmed mean of $M$ values and batch correction with the help of NOISeqBIO (43). Differentially expressed genes were selected with $0.75<$ fold change $>1.5$ and FDR $<0.05$. bigWig files were obtained by the use of BamCoverage from deepTools2 (44) (-bs 20 -smoothLength 40 -p max -normalizeTo1x 2150570000 -e 150). GO biological processes and KEGG pathway analyses from different groups of genes were performed by compareCluster using the enrichGO and enrichKEGG databases with a $P$ value less than 0.05 , with Bonferroni's test as a method to correct the $P$ value.

A heatmap of differentially regulated genes was generated using heatmap. 2 from ggplot in Rstudio. SOX9 ChIP-Seq data used in this manuscript were obtained from GSE69109 (26) and were crossed with our RNA-Seq data by gene name using the setdiff function from R. Our data set of RNA-Seq data is deposited in the National Center for Biotechnology Information's Gene Expression Omnibus database under accession number GSE132265.

Isolation of adult cardiomyocytes and cardiac fibroblast cultures. Adult ventricular cardiomyocytes were prepared from uninjured adult mice as previously described using a Langendorff system (45).

Cardiac fibroblasts were isolated from 3- to 8-week-old Sox $9^{f / f l}$ mice by cutting the whole heart without atria quickly into small pieces in ice-cold PBS. Digestion was performed using Liberase TH (Roche) with SADO buffer mix solution (20 mM HEPES-NaOH [Roth, Nr. 9105] [pH 7,6], $130 \mathrm{mM} \mathrm{NaCl}$ [Roth, Nr. 9265], 3 mM KCl [Roth, Nr. 6781], 1 mM NaH2PO4 [Sigma, Nr. S5011], 4 mM Glucose [Roth, Nr. HN06], 3 mM MgSO 4 [Roth, Nr. 2278] in sterile, filtered $\mathrm{H}_{2} \mathrm{O}$ ). The cardiac fibroblasts were plated and the medium was changed with Dulbecco's modified Eagle medium (DMEM) supplemented with 10\% FBS after 3 hours. Isolated fibroblasts were then infected with AdCre or Ad $\beta$-gal.

For the collagen contraction assay, infected fibroblasts $\left(4 \times 10^{4}\right.$ per well) were poured into collagen type I gel matrices including DMEM, as previously described by others (46). The contraction level was analyzed after 48 hours (ImageJ, NIH) by calculating (gel area at 0 hours - gel area at 48 hours)/gel area at 0 hours $\times 100 \%$. Six replicates were analyzed per condition.

For assessment of proliferation, AdCre-infected and Ad $\beta$-gal-infected fibroblasts $\left(6 \times 10^{3}\right.$ or $12 \times 10^{3}$ per well, respectively) were plated on a 96-well plate. A colorimetric BrdU incorporation immunoassay (Roche) was used following the manufacturer's instructions. The absorbance was measured at $370 \mathrm{~nm}$ every 2 minutes over a period from 0 to 20 minutes. The proliferation assay was repeated 3 times with 8 wells per group in each run.

To analyze the migration capacity, $5 \times 10^{4}$ AdCre-infected or Ad $\beta$-gal-infected fibroblasts were plated per well on 24 -well plates 48 hours after infection. Subsequently, fibroblasts were stimulated with recombinant human TGF- $\beta 1$ (10 ng/mL, Peprotech, 100-21), human angiotensin II (100 nmol/L, Merck, 05-230101-5MG), or 10\% FBS or were left unstimulated. Fibroblast monolayers were then scratched with a 200 $\mu \mathrm{L}$ pipet tip. To analyze the migration of the fibroblasts, the same scratch area was captured with the Axio Observer.Z1 microscope after 0, 4, 16, and 24 hours. Migration ratio was calculated as (cell-free area at 0 $\mathrm{h}$ - cell-free area at 4, 16, or $24 \mathrm{~h}$ )/cell-free area at $0 \mathrm{~h}$, and the cell-free area was quantified using ImageJ. The scratch assay was carried out in 3 biological replicates with 3 to 4 wells per condition.

Statistics. All data are presented as mean \pm SEM. The investigators were blinded for mouse genotype during surgeries, echocardiography, organ weight determination, and all histological and immunofluorescence quantifications. An unpaired, 2-tailed Student's $t$ test was used to determine statistical significance when 2 groups were compared. For comparison of more than 2 groups, statistical significance was determined by using 1-way ANOVA (with Tukey's multiple-comparisons test) for 1 independent variable and 2-way ANOVA (with Holm-Šídák's multiple-comparisons test) for 2 independent variables (GraphPad Prism 7 software). $P$ values less than 0.05 were considered statistically significant.

Study approval. All animal procedures described in this study were approved by the Lower Saxony State Office for Consumer Protection and Food Safety (Wardenberg, Germany). (33.12-42502-04-16/2098). All procedures including the use and care of animals were carried out according to the Guide for the Care and Use of Laboratory Animals published by the National Research Council (National Academies Press, revised 1996) and the German animal protection code. 


\section{Author contributions}

GMS, KK, and JH designed the study, planned all experiments, and analyzed the data. GMS, KK, YW, AG, MH, XW, and RG performed experiments. AK, RK, and SJC provided important reagents and critically revised the manuscript. JC, NF, KCW, GD, and JB gave advice for the project, analyzed data, and critically revised the manuscript. GMS and JH wrote the manuscript. JH supervised the study. All authors read and approved the manuscript.

\section{Acknowledgments}

This study was supported by the Deutsche Forschungsgemeinschaft through the Cluster of Excellence Rebirth (EXC 62/1 and EXC 62/3 to KCW, JB, and JH) and the Heisenberg Program (HE 3658/6-1\&2 to $\mathrm{JH}$ ) along with NIH grant HL148165 (to SJC). GMS received intramural funding from the Hannover Medical School ("Hochschulinterne Leistungsförderung"). KK was supported by a research grant from the German Cardiac Society.

Address correspondence to: Joerg Heineke, Department of Cardiovascular Research, European Center for Angioscience, Medizinische Fakultät Mannheim, Universität Heidelberg, Ludolf-Krehl-Str. 7-11, 68167 Mannheim, Germany. Phone: 49.621.383.71855; Email: joerg.heineke@medma.uni-heidelberg.de.

1. Go AS, et al. Heart disease and stroke statistics - 2013 update: a report from the American Heart Association. Circulation. 2013;127(1):e6-e245.

2. Tarone G, et al. Targeting myocardial remodelling to develop novel therapies for heart failure: a position paper from the Working Group on Myocardial Function of the European Society of Cardiology. Eur J Heart Fail. 2014;16(5):494-508.

3. Kong P, Christia P, Frangogiannis NG. The pathogenesis of cardiac fibrosis. Cell Mol Life Sci. 2014;71(4):549-574.

4. Heineke J, Molkentin JD. Regulation of cardiac hypertrophy by intracellular signalling pathways. Nat Rev Mol Cell Biol. 2006;7(8):589-600.

5. Frangogiannis NG. The extracellular matrix in myocardial injury, repair, and remodeling. J Clin Invest. 2017;127(5):1600-1612.

6. Fraccarollo D, Galuppo P, Bauersachs J. Novel therapeutic approaches to post-infarction remodelling. Cardiovasc Res. 2012;94(2):293-303

7. Burchfield JS, Xie M, Hill JA. Pathological ventricular remodeling: mechanisms: part 1 of 2. Circulation. 2013;128(4):388-400

8. Tallquist MD, Molkentin JD. Redefining the identity of cardiac fibroblasts. Nat Rev Cardiol. 2017;14(8):484-491.

9. Akiyama H, Chaboissier MC, Martin JF, Schedl A, de Crombrugghe B. The transcription factor Sox 9 has essential roles in successive steps of the chondrocyte differentiation pathway and is required for expression of Sox 5 and Sox6. Genes Dev. 2002;16(21):2813-2828.

10. Barrionuevo F, Scherer G. SOX E genes: SOX9 and SOX8 in mammalian testis development. Int J Biochem Cell Biol. 2010;42(3):433-436.

11. Huang B, Wang S, Ning Y, Lamb AN, Bartley J. Autosomal XX sex reversal caused by duplication of SOX9. Am J Med Genet. 1999;87(4):349-353.

12. Lefebvre V, Dumitriu B, Penzo-Méndez A, Han Y, Pallavi B. Control of cell fate and differentiation by Sry-related high-mobility-group box (Sox) transcription factors. Int J Biochem Cell Biol. 2007;39(12):2195-2214

13. Foster JW, et al. Campomelic dysplasia and autosomal sex reversal caused by mutations in an SRY-related gene. Nature. 1994;372(6506):525-530.

14. Wagner T, et al. Autosomal sex reversal and campomelic dysplasia are caused by mutations in and around the SRY-related gene SOX9. Cell. 1994;79(6):1111-1120.

15. Bennett MR, Czech KA, Arend LJ, Witte DP, Devarajan P, Potter SS. Laser capture microdissection-microarray analysis of focal segmental glomerulosclerosis glomeruli. Nephron Exp Nephrol. 2007;107(1):e30-e40.

16. Hanley KP, Oakley F, Sugden S, Wilson DI, Mann DA, Hanley NA. Ectopic SOX9 mediates extracellular matrix deposition characteristic of organ fibrosis. J Biol Chem. 2008;283(20):14063-14071.

17. Peacock JD, Levay AK, Gillaspie DB, Tao G, Lincoln J. Reduced sox9 function promotes heart valve calcification phenotypes in vivo. Circ Res. 2010;106(4):712-719.

18. Lacraz GPA, et al. Tomo-Seq identifies SOX9 as a key regulator of cardiac fibrosis during ischemic injury. Circulation. 2017;136(15):1396-1409.

19. Pinto AR, et al. Revisiting cardiac cellular composition. Circ Res. 2016;118(3):400-409.

20. Lajiness JD, Conway SJ. Origin, development, and differentiation of cardiac fibroblasts. J Mol Cell Cardiol. 2014;70:2-8.

21. Takeda N, et al. Cardiac fibroblasts are essential for the adaptive response of the murine heart to pressure overload. $J$ Clin Invest. 2010;120(1):254-265

22. Kong P, Christia P, Saxena A, Su Y, Frangogiannis NG. Lack of specificity of fibroblast-specific protein 1 in cardiac remodeling and fibrosis. Am J Physiol Heart Circ Physiol. 2013;305(9):H1363-H1372.

23. Ivey MJ, Tallquist MD. Defining the cardiac fibroblast. Circ J. 2016;80(11):2269-2276.

24. Conway SJ, Molkentin JD. Periostin as a heterofunctional regulator of cardiac development and disease. Curr Genomics. 2008;9(8):548-555.

25. Fu X, et al. Specialized fibroblast differentiated states underlie scar formation in the infarcted mouse heart. J Clin Invest. 2018;128(5):2127-2143. 
26. Ohba S, He X, Hojo H, McMahon AP. Distinct transcriptional programs underlie Sox9 regulation of the mammalian chondrocyte. Cell Rep. 2015;12(2):229-243.

27. Frangogiannis NG. The mechanistic basis of infarct healing. Antioxid Redox Signal. 2006;8(11-12):1907-1939.

28. Shinde AV, Frangogiannis NG. Fibroblasts in myocardial infarction: a role in inflammation and repair. J Mol Cell Cardiol. 2014;70:74-82.

29. Lindner D, et al. Cardiac fibroblasts support cardiac inflammation in heart failure. Basic Res Cardiol. 2014;109(5):428.

30. Sato Y, Yanagita M. Resident fibroblasts in the kidney: a major driver of fibrosis and inflammation. Inflamm Regen. 2017;37:17.

31. Kanisicak O, et al. Genetic lineage tracing defines myofibroblast origin and function in the injured heart. Nat Commun. 2016;7:12260.

32. Kamachi Y, Kondoh H. Sox proteins: regulators of cell fate specification and differentiation. Development. 2013;140(20):4129-4144

33. Pritchett J, Athwal V, Roberts N, Hanley NA, Hanley KP. Understanding the role of SOX9 in acquired diseases: lessons from development. Trends Mol Med. 2011;17(3):166-174.

34. Furtado MB, et al. Cardiogenic genes expressed in cardiac fibroblasts contribute to heart development and repair. Circ Res. 2014;114(9):1422-1434.

35. Creemers EE, Cleutjens JP, Smits JF, Daemen MJ. Matrix metalloproteinase inhibition after myocardial infarction: a new approach to prevent heart failure? Circ Res. 2001;89(3):201-210.

36. Fontana L, et al. Fibronectin is required for integrin $\alpha v \beta 6$-mediated activation of latent TGF- $\beta$ complexes containing LTBP-1. FASEB J. 2005;19(13):1798-1808.

37. Ge G, Greenspan DS. BMP1 controls TGF $\beta 1$ activation via cleavage of latent TGF $\beta$-binding protein. J Cell Biol. 2006;175(1):111-120.

38. Ammirante M, Shalapour S, Kang Y, Jamieson CA, Karin M. Tissue injury and hypoxia promote malignant progression of prostate cancer by inducing CXCL13 expression in tumor myofibroblasts. Proc Natl Acad Sci U S A. 2014;111(41):14776-14781.

39. Kist R, Schrewe H, Balling R, Scherer G. Conditional inactivation of Sox9: a mouse model for campomelic dysplasia. Genesis. 2002;32(2):121-123.

40. Lindsley A, et al. Identification and characterization of a novel Schwann and outflow tract endocardial cushion lineage-restricted periostin enhancer. Dev Biol. 2007;307(2):340-355

41. Froese N, et al. Anti-androgenic therapy with finasteride improves cardiac function, attenuates remodeling and reverts patholog ic gene-expression after myocardial infarction in mice. J Mol Cell Cardiol. 2018;122:114-124.

42. Heinz S, et al. Simple combinations of lineage-determining transcription factors prime cis-regulatory elements required for macrophage and B cell identities. Mol Cell. 2010;38(4):576-589.

43. Tarazona S, Furió-Tarí P, Turrà D, Pietro AD, Nueda MJ, Ferrer A, Conesa A. Data quality aware analysis of differential expression in RNA-seq with NOISeq R/Bioc package. Nucleic Acids Res. 2015;43(21):e140.

44. Ramírez F, Dündar F, Diehl S, Grüning BA, Manke T. deepTools: a flexible platform for exploring deep-sequencing data. Nucleic Acids Res. 2014;42(Web Server issue):W187-W191..

45. Appari M, et al. C1q-TNF-related protein-9 promotes cardiac hypertrophy and failure. Circ Res. 2017;120(1):66-77.

46. Davis J, Burr AR, Davis GF, Birnbaumer L, Molkentin JD. A TRPC6-dependent pathway for myofibroblast transdifferentiation and wound healing in vivo. Dev Cell. 2012;23(4):705-715. 